\title{
Studies on the Effect of Establishment Methods and Foliar Nutrition on Productivity of Transplanted Finger Millet (Eleusine coracana) under Irrigated Condition
}

\author{
M. Sivashankar ${ }^{1 *}$, V. K. Paulpandi ${ }^{1}$, R. Durai Singh ${ }^{1}$ and K. Thangaraj ${ }^{2}$ \\ ${ }^{1}$ Department of Agronomy, ${ }^{2}$ Department of Plant Breeding and Genetics, \\ $A C \&$ RI, Madurai, India \\ *Corresponding author
}

\section{A B S T R A C T}

\begin{tabular}{l} 
Ke y w o r d s \\
$\begin{array}{l}\text { Foliar Nutrition, } \\
\text { Finger Millet, } \\
\text { Eleusine coracana }\end{array}$ \\
\hline Article Info \\
\hline $\begin{array}{l}\text { Accepted: } \\
17 \text { September } 2020 \\
\text { Available Online: } \\
10 \text { October } 2020\end{array}$ \\
\hline
\end{tabular}

\section{Introduction}

Finger millet (Eleusine coracana) is one of the most important nutri cereals which possessing remarkable ability to survive under severe drought. This crop is cultivated mainly for the nutritious grains and straw. Ragi straw is good fodder for cattle and used for hay making.It occupies maximum area among the small millets. In India, finger millet is cultivated about $1.19 \mathrm{M}$ hectares with total production of 1.98 Mtonnes and productivity of $1662 \mathrm{~kg} / \mathrm{ha}$. In Tamil Nadu finger millet is cultivated in an area of $0.086 \mathrm{M}$ hectares with total production of 0.32 Mtonnes and productivity of $3714 \mathrm{~kg} / \mathrm{ha}$ respectively (India stat, 2017-2018). The grains can be stored for $8-10$ years and thus, it is an important famine food (Michaelraj and Shanmugam, 2013).Finger millet grains are more nutritious than wheat and rice. The nutritional values per $100 \mathrm{~g}$ of finger millet are: Protein $7.3 \mathrm{~g}$; Minerals $2.7 \mathrm{~g}$; Carbohydrates $72 \mathrm{~g}$; Fat $1.3 \mathrm{~g}$; Calcium $344 \mathrm{mg}$ and Fibre $3.6 \mathrm{~g}$. It also contains Iron $5 \mathrm{mg}$ and Food energy 323-350 $\mathrm{K}$ Cal. The finger millet contains important amino acids viz., isoleucine (4.4 g), leucine $(9.5 \mathrm{~g})$, methionine $(3.1 \mathrm{~g})$ and phenyl alanine $(5.2 \mathrm{~g})$ which are deficient in other starchy meals. Millets also contains B vitamins, 
especially niacin, B6 and folic acid calcium, iron, potassium, magnesium and zinc (Vachanth et al., 2010). Finger millet has largely cultivated as a crop under rainfed conditions and which was raised under irrigated condition as transplanting crop. Finger millet productivity and production is extremely lower than the other cereals because of planting pattern, establishment methods, planting of aged seedling, insufficient irrigation and nutrient management practices etc.,

Foliar nutrition is the one of the methods of nutrient application to the plant and foliar application of nutrient is nothing but applying fertilizer in liquid form foliar application of nutrients are increase the maximum nutrient absorption in plants. It will penetrate in the leaf cuticle and cells which was helpful for rapid growth of the plants (Manonmani and Srimathi, 2009). Shortage of labour, time bound availability and scarcity of resources, especially water are the main causes for delayed transplanting in finger millet. In spite of the labor scarcity, uniform population as well as adequate plant spacing cannot be possible in the field this also one of the factor for low productivity of finger millet. To rectify these problems mechanical transplanting is only the possible way to achieve maximum production and productivity (Vasudevan et al., 2014).

\section{Materials and Methods}

The field experiment was located in field No. 32 of $\mathrm{C}$ block at Agricultural College and Research Institute, Madurai. The experimental site is situated geographically at $9^{\circ} 54^{\prime} \mathrm{N}$ latitude and $78^{\circ} 54^{\prime} \mathrm{E}$ longitude with an altitude of $147 \mathrm{~m}$ above the mean sea level under southern agro-climatic zone of Tamil Nadu. Minimum and Maximum temperature, sunshine hours, relative humidity, wind velocity rainfall and evaporation data were collected from agro meteorological observatory at Agricultural College and Research Institute, Madurai, Tamil Nadu. Experiment field contain available N $(245 \mathrm{~kg}$ ha $^{-1}$ ), available $\mathrm{P}_{2} \mathrm{O}_{5}\left(16.3 \mathrm{~kg} \mathrm{ha}^{-1}\right.$, available $\mathrm{K}_{2} \mathrm{O}\left(275 \mathrm{~kg} \mathrm{ha}^{-1}\right)$ and organic carbon $(0.56$ $\%)$.

The trial was laid out in split plot design with three replications. The treatments at main plots consisted of different methods of transplanting viz., normal transplanting (30 $\mathrm{cm} \times 10 \mathrm{~cm})\left(\mathrm{M}_{1}\right)$, square transplanting $(17.5$ $\mathrm{cm} \quad \mathrm{x} \quad 17.5 \mathrm{~cm})\left(\mathrm{M}_{2}\right)$ and machine transplanting $(30 \mathrm{~cm} \times 10 \mathrm{~cm})\left(\mathrm{M}_{3}\right)$. In sub plots, different foliar nutrition management practices viz., Panchakavya $(3 \%)\left(\mathrm{S}_{1}\right), \mathrm{PPFM}$ (1\%) $\left(\mathrm{S}_{2}\right)$, Salicylic acid $(40 \mathrm{ppm})\left(\mathrm{S}_{3}\right)$, Humic acid $(2 \%)\left(\mathrm{S}_{4}\right)$ these are chemicals are sprayed in the interval of 25 DAT and 50 DAT.

\section{Results and Discussion}

\section{Plant height}

The total height of the plant was significantly affected by different types of transplanting and foliar nutrition practices. Increased plant height $50.86,64.16,91.68$ and $113.81 \mathrm{~cm}$ at growth stage of 20,40,60 DAT and harvest was noticed in machine transplanting $\left(\mathrm{M}_{3}\right)$ which was followed by manual transplanting 49.83,62.69,92.07 and $109.64 \mathrm{~cm}$ and lower plant height was recorded $46.4, \quad 58.54,84.74$ and $103.62 \mathrm{~cm}$. Mudalagiriyappa et al., (2015) also found similar results in the plant height of the finger millet. with regard to foliar application humic acid $2 \%$ spray $\left(\mathrm{S}_{4}\right)$ at 25 DAT and 50 DAT was recorded maximum plant height 54.85, 70.32101 .20 and118.46 $\mathrm{cm}$ which was followed by salicylic acid at the rate of $40 \mathrm{ppm}$ recorded (49.71 62.67, $91.66112 .98 \mathrm{~cm}$ ) with regard to interaction different types of establishment pattern along with foliar application shown non significance between each other (Table 1). 
Table.1 Effect of different types of transplanting and foliar nutrition management practices on plant height $(\mathrm{cm})$ of Finger millet at 20, 40, 60 DAT and harvest stage

\begin{tabular}{|c|c|c|c|c|c|c|c|c|c|c|c|c|c|c|c|c|}
\hline \multirow[t]{2}{*}{ Treatments } & \multicolumn{3}{|c|}{20 DAT } & \multirow[t]{2}{*}{ Mean } & \multicolumn{3}{|c|}{40 DAT } & \multirow[t]{2}{*}{ Mean } & \multicolumn{3}{|c|}{60 DAT } & \multirow[t]{2}{*}{ Mean } & \multicolumn{3}{|c|}{ Harvest } & \multirow[b]{2}{*}{ Mean } \\
\hline & $\mathbf{M}_{1}$ & $\mathbf{M}_{2}$ & $\mathbf{M}_{3}$ & & $\mathbf{M}_{1}$ & $\mathbf{M}_{2}$ & $\mathbf{M}_{3}$ & & $\mathbf{M}_{1}$ & $\mathbf{M}_{2}$ & $\mathbf{M}_{3}$ & & $\mathbf{M}_{1}$ & $\mathbf{M}_{2}$ & $\mathbf{M}_{3}$ & \\
\hline $\mathbf{S}_{1}$ & 43.91 & 39.04 & 46.13 & 43.03 & 53.00 & 50.63 & 55.61 & 53.08 & 80.23 & 75.16 & 80.65 & 78.68 & 94.46 & 79.93 & 106.36 & 93.58 \\
\hline $\mathbf{S}_{2}$ & 49.87 & 47.04 & 48.93 & 48.61 & 63.08 & 57.93 & 62.34 & 61.12 & 90.32 & 81.67 & 87.30 & 86.43 & 113.28 & 107.62 & 112.33 & 111.08 \\
\hline $\mathbf{S}_{3}$ & 50.09 & 47.66 & 51.36 & 49.71 & 63.25 & 58.96 & 65.80 & 62.67 & 94.31 & 85.56 & 95.12 & 91.66 & 114.16 & 109.75 & 115.02 & 112.98 \\
\hline$S_{4}$ & 55.45 & 52.10 & 57.01 & 54.85 & 71.43 & 66.63 & 72.90 & 70.32 & 103.42 & 96.55 & 103.63 & 101.20 & 116.67 & 117.17 & 121.54 & 118.46 \\
\hline \multirow[t]{2}{*}{ Mean } & 49.83 & 46.46 & 50.86 & & 62.69 & 58.54 & 64.16 & & 92.07 & 84.74 & 91.68 & & 109.64 & 103.62 & 113.81 & \\
\hline & $\mathbf{M}$ & $\mathbf{S}$ & $\mathbf{M} \times \mathbf{S}$ & $\mathbf{S} \times \mathbf{M}$ & $\mathbf{M}$ & $\mathbf{S}$ & $\mathbf{M} \times \mathbf{S}$ & $\mathbf{S} \times \mathbf{M}$ & $\mathbf{M}$ & $\mathbf{S}$ & $\mathbf{M} \times \mathbf{S}$ & $\mathbf{S} \times \mathbf{M}$ & $\mathbf{M}$ & $\mathbf{S}$ & M X S & SX M \\
\hline $\mathrm{SE}(\mathrm{d})$ & 1.22 & 1.70 & 2.41 & 2.41 & 1.56 & 2.91 & 4.11 & 4.11 & 2.20 & 3.73 & 5.27 & 5.27 & 2.72 & 5.05 & 7.14 & 7.14 \\
\hline $\mathrm{CD}(\mathrm{P}=\mathbf{0 . 0 5 \% )}$ & 2.86 & 3.63 & NS & NS & 3.67 & 6.20 & NS & NS & 5.17 & 7.95 & NS & NS & 6.39 & 10.76 & NS & NS \\
\hline
\end{tabular}

Table.2 Effect of different types of transplanting and foliar nutrition management practices on LAI of finger millet at 20, 60, 40 DAT and at harvest stage

\begin{tabular}{|c|c|c|c|c|c|c|c|c|c|c|c|c|}
\hline \multirow[t]{2}{*}{ Treatments } & \multicolumn{3}{|c|}{20 DAT } & \multirow[t]{2}{*}{ Mean } & \multicolumn{3}{|c|}{40 DAT } & \multirow[t]{2}{*}{ Mean } & \multicolumn{3}{|c|}{60 DAT } & \multirow[b]{2}{*}{ Mean } \\
\hline & $\mathbf{M}_{1}$ & $\mathbf{M}_{2}$ & $\mathbf{M}_{3}$ & & $\mathbf{M}_{1}$ & $\mathbf{M}_{2}$ & $\mathbf{M}_{3}$ & & $\mathbf{M}_{1}$ & $\mathbf{M}_{2}$ & $\mathbf{M}_{3}$ & \\
\hline $\mathbf{S}_{1}$ & 1.84 & 1.65 & 1.92 & 1.80 & 2.46 & 2.23 & 2.66 & 2.45 & 3.08 & 2.85 & 3.21 & 3.05 \\
\hline $\mathbf{S}_{2}$ & 2.20 & 1.97 & 2.13 & 2.10 & 2.32 & 2.86 & 3.20 & 2.79 & 3.96 & 3.52 & 3.82 & 3.77 \\
\hline $\mathbf{S}_{\mathbf{3}}$ & 2.26 & 2.04 & 2.40 & 2.23 & 3.48 & 3.16 & 3.64 & 3.43 & 4.10 & 3.66 & 4.20 & 3.99 \\
\hline $\mathbf{S}_{4}$ & 2.72 & 2.53 & 2.78 & 2.68 & 3.57 & 3.72 & 3.73 & 3.67 & 4.46 & 4.38 & 4.52 & 4.45 \\
\hline \multirow[t]{2}{*}{ Mean } & 2.26 & 2.05 & 2.31 & & 2.96 & 2.99 & 3.31 & & 3.90 & 3.60 & 3.94 & \\
\hline & M & $\mathbf{S}$ & $\mathbf{M} \times \mathbf{S}$ & $\mathbf{S} \times \mathbf{M}$ & $\mathbf{M}$ & $\mathbf{S}$ & $\mathbf{M} \times \mathbf{S}$ & $\mathbf{S} \times \mathbf{M}$ & $\mathbf{M}$ & $\mathbf{S}$ & $\mathbf{M} \times \mathbf{S}$ & $\mathbf{S} \times \mathbf{M}$ \\
\hline $\mathrm{SE}(\mathrm{d})$ & 0.06 & 0.10 & 0.14 & 0.14 & 0.08 & 0.17 & 0.24 & 0.24 & 0.09 & 0.17 & 0.24 & 0.24 \\
\hline $\begin{array}{c}C D \\
(P=0.05 \%)\end{array}$ & 0.15 & 0.21 & NS & NS & 0.19 & 0.36 & NS & NS & 0.22 & 0.36 & NS & NS \\
\hline
\end{tabular}


Table.3 Effect of different types of transplanting and foliar nutrition management practices on CGR (Kg/ha) of finger millet at 20, 60, 40 DAT and at harvest stage

\begin{tabular}{|c|c|c|c|c|c|c|c|c|c|c|c|c|c|c|c|c|}
\hline \multirow[t]{2}{*}{ Treatments } & \multicolumn{3}{|c|}{$0-20$} & \multirow[t]{2}{*}{ Mean } & \multicolumn{3}{|c|}{$20-40$} & \multirow[t]{2}{*}{ Mean } & \multicolumn{3}{|c|}{$40-60$} & \multirow[t]{2}{*}{ Mean } & \multicolumn{3}{|c|}{ 60-Harvest } & \multirow[b]{2}{*}{ Mean } \\
\hline & $\mathbf{M}_{1}$ & $\mathbf{M}_{2}$ & $\mathbf{M}_{3}$ & & $\mathbf{M}_{1}$ & $\mathbf{M}_{2}$ & $\mathbf{M}_{3}$ & & $\mathbf{M}_{1}$ & $\mathbf{M}_{2}$ & $\mathbf{M}_{3}$ & & $\mathbf{M}_{1}$ & $\mathbf{M}_{2}$ & $\mathbf{M}_{3}$ & \\
\hline$S_{1}$ & 30.10 & 27.82 & 43.26 & 33.73 & 49.37 & 45.50 & 51.18 & 48.68 & 66.80 & 60.26 & 70.13 & 65.73 & 86.13 & 71.22 & 91.43 & 82.93 \\
\hline $\mathbf{S}_{2}$ & 37.02 & 43.88 & 33.46 & 38.12 & 56.87 & 53.10 & 55.67 & 55.21 & 80.60 & 73.42 & 78.36 & 77.46 & 103.80 & 95.20 & 101.56 & 100.19 \\
\hline $\mathbf{S}_{3}$ & 38.86 & 35.26 & 40.06 & 38.06 & 57.36 & 54.20 & 57.36 & 56.31 & 82.13 & 76.23 & 84.65 & 81.00 & 105.36 & 98.70 & 108.43 & 104.16 \\
\hline $\mathbf{S}_{4}$ & 43.77 & 36.25 & 44.15 & 41.39 & 60.73 & 57.59 & 65.93 & 61.42 & 86.79 & 85.10 & 90.23 & 87.37 & 111.90 & 110.12 & 116.17 & 112.73 \\
\hline \multirow[t]{2}{*}{ Mean } & 37.44 & 35.80 & 40.23 & & 56.08 & 52.60 & 57.53 & & 79.08 & 73.75 & 80.84 & & 101.80 & 93.81 & 104.40 & \\
\hline & $\mathbf{M}$ & $\mathbf{S}$ & $\mathbf{M} \times \mathbf{S}$ & $\mathbf{S} \times \mathbf{M}$ & $\mathbf{M}$ & $\mathbf{S}$ & $\mathbf{M} \times \mathbf{S}$ & $\mathbf{S} \times \mathbf{M}$ & M & $\mathbf{S}$ & $\mathbf{M} \times \mathbf{S}$ & $\mathbf{S} \times \mathbf{M}$ & $\mathbf{M}$ & $\mathbf{S}$ & MXS & SX M \\
\hline $\operatorname{SE}(d)$ & 0.94 & 1.13 & 1.94 & 1.96 & 1.36 & 2.15 & 3.51 & 3.74 & 1.94 & 2.38 & 4.07 & 4.12 & 2.64 & 2.83 & 5.00 & 4.91 \\
\hline $\mathrm{CD}(\mathrm{P}=0.05 \%)$ & 2.63 & 2.38 & NS & NS & 3.78 & 4.53 & NS & NS & 5.40 & 5.00 & NS & NS & 7.34 & 5.95 & NS & NS \\
\hline
\end{tabular}




\section{Leaf Area Index}

The leaf area index (LAI) is determined by the number of tillers, number of green leaves hill $^{-1}$ and average leaf size (Rai and Murty, 1976). Leaf area index was moderately affected by transplanting methods and foliar application practices. machine transplanting shown better variance in its growth stage $(2.31,3.31,3.94)$ which was on par with conventional transplanting $(2.26,2.99,3.60)$ which was followed by square transplanting. These results are in close conformity with findings of Anitha et al., (2017) and Amin and Haque (2009) (Table 2).

With regard to foliar application $\left(\mathrm{S}_{4}\right)$ humic acid 2\% foliar application was recorded maximum leaf area index $(2.68,3.67,4.45)$ which was followed by salicylic acid $40 \mathrm{ppm}$ on 25 DAT and 50 DAT was recorded $(2.23,3.43,3.99)$ and lower leaf area index was recorded in panchagavya $3 \%$ foliar application $(1.80,2.45,3.05)$ with regard to interaction, there is no significance was recorded in leaf area index parameter.

\section{Crop Growth Rate}

CGR was significantly affected by different types of transplanting and foliar application methods. maximum crop growth rate was recorded at 20, 40,60 DAT and harvest stage $(40.23,57.53,80.84,104.40 \mathrm{Kg} / \mathrm{ha})$ in machine transplanting $\left(\mathrm{M}_{3)}\right.$ lower crop growth rate was reported in conventional transplanting $\left(\mathrm{M}_{1)} 37.44,56.08,79.08,101.80\right.$ $(\mathrm{Kg} / \mathrm{ha})$ with regard to foliar application practices, $\left(\mathrm{S}_{4}\right)$ humic acid $2 \%$ foliar application in 25 DAT and 50 DAT was recorded that increased CGR $(33.73,61.42$, $87.37,112.73 \mathrm{Kg} / \mathrm{ha}$ ) which was followed by salicylic acid $40 \mathrm{ppm}\left[\left(\mathrm{S}_{3)}(38.06,56.31,81\right.\right.$, $104.16 \mathrm{Kg} / \mathrm{ha}$ ] with regard to interaction, there is no significance was recorded in crop growth rate (Table 3 ).
In conclusion by adopting machine transplanting along with humic acid 2\% foliar application on 25 DAT and 45 DAT (vegetative and flowering stage) is registered maximum plant height, LAI, CGR.

\section{References}

Anitha, D., V. Nagavani, and V. Chandrika. 2017. "Influence of crop geometry and age of seedlings on yield, nutrient uptake, postharvest nutrient status and economics of finger millet." Green Farming 8 (1): 160163.

Amin, AKMK, and M AminulHaque. 2009. "Seedling age influence rice (Oryza sativa L.) performance." Philippine Journal of Science 138 (2): 219-226.

Manonmani, V., \&Srimathi, P. (2009). Influence of mother crop nutrition on seed yield and quality of blackgram. Madras Agricultural Journal, 96(1-6), 125-128.

Mudalagiriyappa, B, BK Ramachandrappa, and HV Nanjappa. 2015. "Influence of customized fertilizers on growth and yield of Finger Millet \{Eleusinecoracana $(\mathrm{L}$. Gaertn.\} inAlfisols of Southern India." Indian J. Dryland Agric. Res. \&Dev30 (1): 50-5

Rai, R. S. U. and K. S. Murty. 1976. Note on the effect of partial submergence of plant on growth and yield in early high yielding rice varieties. Ind. J. Agric. Res., 10: 261264

Vachanth, M.C., K.M. SubbuRathinam, R. Preethi and M. Loganathan. 2010. Controlled atmoshpheric storage techniques for safe storage

processed little millet. Acad. J. Entomol., 3(1): 13-16.

Vasudevan, S., R.C.M. Basangouda, S. Doddagoudar, and N. Shakuntala. 2014. "Standardization of Seedling Characteristics for Paddy Transplanter." Journal of Advanced Agricultural Technologies Vol. 1 (2). 


\section{How to cite this article:}

Sivashankar, M., V. K. Paulpandi, R. Durai Singh and Thangaraj, K. 2020. Studies on the Effect of Establishment Methods and Foliar Nutrition on Productivity of Transplanted Finger Millet (Eleusine coracana) under Irrigated Condition. Int.J.Curr.Microbiol.App.Sci. 9(10): 2446-2451. doi: https://doi.org/10.20546/ijcmas.2020.910.292 